\title{
Influence of the sign of the coupling on the temperature dependence of optical properties of one-dimensional exciton models
}

\author{
L Cruzeiro \\ CCMAR and FCT, Universidade of Algarve, Campus de Gambelas, 8005-139 Faro, Portugal
}

Received 12 June 2008, in final form 24 July 2008

Published 10 September 2008

Online at stacks.iop.org/JPhysB/41/195401

\begin{abstract}
A new physical cause for a temperature-dependent double peak in exciton systems is put forward within a thermal equilibrium approach for the calculation of optical properties of exciton systems. Indeed, it is found that one-dimensional exciton systems with only one molecule per unit cell can have an absorption spectrum characterized by a double peak provided that the coupling between excitations in different molecules is positive. The two peaks, whose relative intensities vary with temperature, are located around the exciton band edges, being separated by an energy of approximately $4 V$, where $V$ is the average coupling between nearest neighbours. For small amounts of diagonal and off-diagonal disorder, the contributions from the intermediate states in the band are also visible as intermediate structure between the two peaks, this being enhanced for systems with periodic boundary conditions. At a qualitative level, these results correlate well with experimental observations in the molecular aggregates of the thiacarbocyanine dye THIATS and in the organic crystals of acetanilide and $\mathrm{N}$-methylacetamide.
\end{abstract}

\section{Introduction}

One-dimensional exciton models were first developed to describe the electronic properties of crystals [1], but they have since been applied to the collective electronic and vibrational excitations of a large variety of systems from organic crystals [2-6] to J- and H-aggregates [7-14] and to proteins [15-21]. An important parameter in the exciton models is the coupling between excitations at different sites which, in many cases, arises from dipole-dipole interactions where the dipoles can be treated as point dipoles. Although in most systems this term is negative, there are cases in which it can be positive as well $[6,12,13,17,20]$. The electronic or vibrational states, however, do not depend on the sign of the coupling and even if a few studies have considered positive couplings $[6,12,13,17,20]$, to the author's best knowledge, the effect that the sign of the coupling parameter can have on the temperature dependence of optical properties has not been fully appreciated. Indeed, theoretical studies are usually done for systems with negative couplings [4, 5, 7, 10-12] and although positive couplings arise when the geometries of specific systems are taken into account $[6,12,13,17,20]$, the effect that those positive couplings alone can have on the temperature dependence of spectral properties has not been discussed.

On the other hand, the variation of optical properties with temperature has been the object of many theoretical and experimental studies [7-12]. In particular, the dynamics of excitons, at a finite temperature and for both negative and positive couplings, has been modelled in a number of recent papers in which intraband transitions, due to the coupling of excitons to lattice vibrations, are described by a Pauli master equation in terms of the microscopic rates for those transitions [10-14]. While in this latter approach, which describes the non-equilibrium relaxation that follows the initial absorption of radiation, the physical influence of the sign of the coupling is buried within the calculation of the transition rates from which the linewidths are obtained, in the approach proposed here, which is valid when the emission spectrum is approximately equal to the absorption spectrum, the interplay between the sign of the coupling and the thermal equilibrium population of excited states will be considered explicitly. Expressions 